\title{
Transient bilateral vocal cord paralysis after endotracheal intubation with double-lumen tube
} -A case report-

\author{
Dae Myoung Jeong ${ }^{1}$, Gunn Hee Kim² ${ }^{2}$ Jie Ae Kim ${ }^{1}$, and Sangmin Maria Lee ${ }^{1}$ \\ Department of Anesthesiology and Pain Medicine, 'Samsung Medical Center, Sungkyunkwan University School of Medicine, \\ ${ }^{2}$ National Medical Center, Seoul, Korea
}

Vocal cord paralysis is one of the most serious anesthetic complications related to endotracheal intubation. The practitioner should take extreme care, as bilateral vocal cord paralysis can obstruct the airway and lead to disastrous respiratory problems. There have been many papers on bilateral vocal cord paralysis after neck surgery, but reports on such a condition after lung surgery are very rare. We report a case of bilateral vocal cord paralysis detected after removal of a double-lumen endotracheal tube in a 67-year-old patient who underwent wedge resection by videoassisted thoracoscopic surgery. We also note that he recovered spontaneously without complications within a day. (Korean J Anesthesiol 2010; 59: S9-S12)

Key Words: Bilateral vocal cord paralysis, Double-lumen endotracheal tube, Postoperative stridor.

Bilateral vocal cord paralysis is widely known to be a complication of general anesthesia [1,2]. There have been few reports, however, of bilateral vocal cord paralysis related to a double-lumen endotracheal tube (DLT) [3].

We encountered a patient with bilateral vocal cord paralysis, detected immediately after extubation, which resolved one day after wedge resection by video-assisted thoracoscopic surgery (VATS) of the left upper lobe of the lung. We report this event and include other relevant studies and information.

\section{Case Report}

A 67-year-old man, $164.5 \mathrm{~cm}$ in height and $64.7 \mathrm{~kg}$ in weight, was admitted to the hospital for investigation of abnormal shadows on chest X-ray in both lungs. Approximately 3 months before admission, he was diagnosed with pancreatic ductal adenocarcinoma and underwent surgery, which consisted of a distal pancreatectomy, splenectomy, and left adrenalectomy. The patient did not have hypertension, diabetes mellitus, cardiovascular disease or other associated disorder. No

Received: October 23, 2009. Revised: 1st, November 9, 2009; 2nd, November 17, 2009. Accepted: December 18, 2009.

Corresponding author: Gunn Hee Kim, M.D., Department of Anesthesiology and Pain Medicine, National Medical Center, 17-79, Euljiro 6-ga, Jung-gu, Seoul 100-799, Korea. Tel: 82-2-2260-7372, Fax: 82-2-2262-4766, E-mail: ipsse@dreamwiz.com

(c) This is an open-access article distributed under the terms of the Creative Commons Attribution Non-Commercial License (http:// creativecommons.org/licenses/by-nc/3.0/), which permits unrestricted non-commercial use, distribution, and reproduction in any medium, provided the original work is properly cited. 
abnormal findings were detected in the preoperative physical examination, electrocardiography, pulmonary function test or echocardiography. On admission, he had no symptoms related to respiration or speech. His chest X-ray and computed tomography scan revealed multiple pulmonary small nodules in both lungs. Hematogenous pulmonary metastasis of pancreatic ductal carcinoma was highly suspected, and pulmonary wedge resection of the left upper lobe by VATS was scheduled for tissue confirmation.

Surgery was performed under general anesthesia using a DLT (Bronchocath left 37F; Mallinckrodt Medical, Athlone, Ireland). We confirmed indirectly that the endotracheal and endobronchial cuffs were not overinflated by palpation of the pilot balloon. The correct placement of the tube was confirmed by fiberoptic bronchoscopy (FOB). After the patient's position was changed to right lateral decubitus, the inflation of the endotracheal and endobronchial cuffs were reconfirmed as above, and the placement of the tube was again confirmed by FOB. We also confirmed that the cuffs were not overinflated before and after one-lung ventilation. Anesthesia was maintained with $2-3 \%$ isoflurane with $\mathrm{O}_{2}$ and air, $1 \mathrm{~L} / \mathrm{min}$ each during two-lung ventilation. One-lung ventilation was provided in volume control mode with $400 \mathrm{ml}$ tidal volume. The $\mathrm{FiO}_{2}$ was 1.0 , respiratory rate was 12 breaths/min and peak airway pressure was maintained at less than $18 \mathrm{cmH}_{2} \mathrm{O}$. The left upper lobe wedge resection was performed without complications. During the operation, the surgeon confirmed that the left recurrent laryngeal nerve was left untouched and remained preserved. The operation time was approximately 1 hour.

At the end of the surgery, glycopyrrolate $0.4 \mathrm{mg}$ and pyridostigmine $15 \mathrm{mg}$ were given after confirming 4 twitches in trainof-four (TOF) stimulation of the ulnar nerve. The tidal volume was $150-250 \mathrm{ml}$, and the patient was able to open his eyes following a verbal order, so extubation was performed. Severe stridor was immediately heard and the patient became irritable. The TOF ratio was greater than 0.9 at that time. Thinking this was due to upper airway spasm following the stress of extubation or vocal cord edema, we applied a jaw thrust maneuver with $10 \mathrm{~L} / \mathrm{min}$ of oxygen via facial mask. The tidal volume was checked and was half of the pre-extubation level. Both lung sounds were decreased, but the peripheral arterial oxygen saturation by pulse oximetry $\left(\mathrm{SpO}_{2}\right)$ was $100 \%$. A salbutamol sulfate puff was applied twice. In addition, solumedrol 125 $\mathrm{mg}$ and dexamethasone $10 \mathrm{mg}$ were intravenously injected. Five minutes later, the stridor had not abated, but the patient seemed to breathe well without the jaw thrust maneuver, so he was transferred to the postanesthesia care unit (PACU) without reintubation.

In the PACU, an electrocardiogram, non-invasive blood pressure, and $\mathrm{SpO}_{2}$ were monitored, and $10 \mathrm{~L} / \mathrm{min}_{2}$ was applied to the patient via facial mask. Severe stridor developed, however, although the $\mathrm{SpO}_{2}$ remained at $100 \%$. We began to suspect that the patient might have other reasons besides laryngeal spasm or vocal cord edema for his stridor. An otolaryngologist examined the patient with a fiberoptic nasopharyngolaryngoscopy and diagnosed him with bilateral vocal cord paralysis of median type with a $1 \mathrm{~mm}$ gap. The otolaryngologist also observed that the vocal cords were not edematous. We planned a tracheostomy, but an arterial blood gas analysis showed mild hypercapnia and normal arterial oxygen pressure $\left(\mathrm{PaCO}_{2} 54\right.$ $\mathrm{mmHg}, \mathrm{PaO}_{2} 223 \mathrm{mmHg}$ ), and the surgeon persistently opposed any invasive procedure. We decided to monitor him in the intensive care unit (ICU), keeping in mind the possibility of an emergency tracheostomy.

In the ICU, $1 \mathrm{mg}$ of midazolam was given intravenously by a pulmonologist, based on his experience with previous patients. After the midazolam injection, the patient became sedated and the stridor diminished. Two hours later, the patient became fully awake and was able to say "Ah". The stridor had completely vanished three and a half hours after extubation. The otolaryngologist's laryngoscope examination was again performed. Bilateral vocal cord paralysis was present, however, and identical to the patient's last examination. The next day, inspection of the vocal cords was again performed and revealed fully recovered vocal cord movement. Thereafter, the patient was transferred to the general ward and discharged six days after surgery.

\section{Discussion}

This case represents bilateral vocal cord paralysis associated with a DLT. To date, many reports have been published about unilateral or bilateral vocal cord paralysis after general anesthesia $[1,2]$, but it is uncommon for paralysis to occur in patients who have undergone lung surgery with a DLT [3].

In this case, we could suspect two possible causes for the paralysis: first, recurrent laryngeal nerve injury during the operation, and second, recurrent laryngeal nerve injury occurring due to intubation with a DLT.

We can rule out the surgery-related injury, because the surgeon did not perform a sternotomy and manipulate the nearby origin of the internal thoracic artery, which is where the left vagus nerve passes.

Ellis and Pallister [4] proposed that compression injury by an overinflated cuff within the larynx could be inflicted on the anterior branch of the recurrent laryngeal nerve passing the medial side of the thyroid lamina. We fixed a DLT at $32 \mathrm{~cm}$ from the incisor and reconfirmed the location of the cuff by using FOB. For this reason, the probability of cuff inflation within the larynx can be ruled out. 
Kikura et al. [5] analyzed the risk factors of vocal cord paralysis and found that vocal cord paralysis occurred more often in conditions that included older patients, patients with hypertension or diabetes mellitus, and increased intubation time. They suggested that aged patients have fragile tissues in the laryngeal system that are more susceptible to mechanical damage, and these tissues could be easily injured by cuff pressure and the tracheal tube itself.

The size of tube has also been correlated with the incidence of postoperative laryngeal injury [6-8]. Published data of Stout et al. [6] imply that the incidence of postoperative hoarseness and vocal cord injury might be directly correlated with the size of the endotracheal tube used. Knoll et al. [7] proposed that a DLT could harm a patient due to its curved endobronchial lumen during intubation as well as extubation. In their study about the usefulness of prophylactic dexamethasone for sore throat and hoarseness after tracheal extubation with a DLT, Park et al. [8] explained that one of the reasons for their finding of increased severity of sore throat according to time was structural injuries in the vocal cords from the DLT. The outer diameter of a 37F DLT is a little larger than that of the $8 \mathrm{~mm}$ single-lumen tube, which corresponds approximately with that of a 35F DLT. Therefore, we can hypothesize that the tissues of aged patients are susceptible to microcirculatory insufficiency, and compression injury by a large-sized and curved DLT could occur more easily. Furthermore, because the extubation was done while the patient's respiratory drive was recovering and because we could not observe the vocal cords during extubation, we can predict that the vocal cord injury could have occurred more easily during extubation.

In this case, the recovery time was shorter than that of other reports. It is known that the usual recovery time of vocal cord paralysis is three months but can vary between six weeks to one year $[5,9,10]$. There has been no report of such a short recovery time as in this case, when vocal cord paralysis developed after endotracheal intubation. There have been several reports, however, about transient bilateral vocal cord paralysis associated with laryngeal mask airway $[11,12]$; in those reports, the paralysis was diagnosed as recurrent laryngeal nerve neuropraxia, a transient episode of motor paralysis with little or no sensory or autonomic dysfunction. The quick recovery in this case suggests a neuropraxia of the recurrent laryngeal nerve as the most likely diagnosis.

We should also rule out the possibility of laryngospasm. Laryngospasm is a reflex closure of the upper airway from spasm of the glottic musculature. During laryngospasm, the false cords and epiglottic body come firmly together. There is no airflow or vocal sound, and the true vocal cords cannot be seen [13]. We confirmed the fixation of the true vocal cords with a laryngoscope, so laryngospasm could be ruled out.
Despite the possibility of quick recovery, if bilateral vocal cord paralysis is diagnosed by a laryngoscope, tracheostomy or transient endotracheal intubation would be the correct treatments. In this case, midazolam $1 \mathrm{mg}$ injected by a pulmonologist was based on the experience that low dose midazolam had diminished dyspnea in patients who had undergone examination with a rigid bronchoscope. Midazolam administration can cause unexpected respiratory failure, so it should have been administered more carefully. In addition, we did not apply continuous positive airway pressure, because it was not available at the time.

In this report, a case of bilateral vocal cord paralysis after wedge resection of the lung is described. The increased susceptibility of tissue to damage in older patients and the use of a DLT have been identified as possible causes of the paralysis. When faced with stridor in a patient who has undergone lung surgery using a DLT, bilateral vocal cord paralysis should be considered as an etiology, and a prompt fiberoptic laryngoscopic examination should be done.

\section{References}

1. Wason R, Gupta P, Gogia AR. Bilateral adductor vocal cord paresis following endotracheal intubation for general anaesthesia. Anaesth Intensive Care 2004; 32: 417-8.

2. Christopher K, Arbelaez C, Yodice PC. Bilateral vocal cord dysfunction complicating short-term intubation and the utility of heliox. Respiration 2002; 69: 366-8.

3. Sagawa M, Donjo T, Isobe T, Notake S, Nakai M, Sugita M, et al. Bilateral vocal cord paralysis after lung cancer surgery with a double-lumen endotracheal tube: a life-threatening complication. J Cardiothorac Vasc Anesth 2006; 20: 225-6.

4. Ellis PD, Pallister WK. Recurrent laryngeal nerve palsy and endotracheal intubation. J Laryngol Otol 1975; 89: 823-6.

5. Kikura M, Suzuki K, Itagaki T, Takada T, Sato S. Age and comorbidity as risk factors for vocal cord paralysis associated with tracheal intubation. Br J Anaesth 2007; 98: 524-30.

6. Stout DM, Bishop MJ, Dwersteg JF, Cullen BF. Correlation of endotracheal tube size with sore throat and hoarseness following general anesthesia. Anesthesiology 1987; 67: 419-21.

7. Knoll H, Ziegeler S, Schreiber JU, Buchinger H, Bialas P, Semyonov $\mathrm{K}$, et al. Airway injuries after one-lung ventilation: a comparison between double-lumen tube and endobronchial blocker: a randomized, prospective, controlled trial. Anesthesiology 2006; 105: 471-7.

8. Park SH, Han SH, Do SH, Kim JW, Rhee KY, Kim JH. Prophylactic dexamethasone decreases the incidence of sore throat and hoarseness after tracheal extubation with a double-lumen endobronchial tube. Anesth Analg 2008; 107: 1814-8.

9. Mun CS, Kim HT, Heo HE, Lee JH, Kwon YE. Unilateral vocal cord palsy occurred after difficult endotracheal intubation using intubating laryngeal mask airway -A case report-. Korean J Anesthesiol 2009; 56: 200-3.

10. Jo EH, Choi YH, Cho IC, Park YC. Unilateral vocal cord paralysis following tracheal extubation -A case report-. Korean J Anesthesiol 
1997; 33: 788-91.

11. Inomata S, Nishikawa T, Suga A, Yamashita S. Transient bilateral vocal cord paralysis after insertion of a laryngeal mask airway. Anesthesiology 1995; 82: 787-8.

12. Sacks MD, Marsh D. Bilateral recurrent laryngeal nerve neuropraxia following laryngeal mask insertion: a rare cause of serious upper airway morbidity. Paediatr Anaesth 2000; 10: 435-7.

13. Donlon JV, Doyle DJ, Feldman MA. Anesthesia for eye, ear, nose, and throat surgery. In: Miller's anesthesia. 6th ed. Edited by Miller DR, Fleisher LA, Johns RA, Savarese JJ, Wiener-Kronish JP, Young WL: Philadelphia, Elsevier Churchill Livingstone. 2005, p 2538. 